\title{
Review
}

\section{Binding of bacterial secondary messenger molecule c di-GMP is a STING operation}

\author{
Neil Shaw ${ }^{1,2}$, Songying Ouyang ${ }^{2}$, Zhi-Jie Liü ${ }^{2 \varpi}$ \\ ${ }^{1}$ Tianjin Key Laboratory of Protein Science, School of Life Sciences, Nankai University, Tianjin 300071, China \\ ${ }^{2}$ National Laboratory of Biomacromolecules, Institute of Biophysics, Chinese Academy of Sciences, Beijing 100101, China \\ $\bowtie$ Correspondence: zjliu@ibp.ac.cn \\ Received July 30, 2012 Accepted December 3, 2012
}

\begin{abstract}
Initial skirmishes between the host and pathogen result in spillage of the contents of the bacterial cell. Amongst the spillage, the secondary messenger molecule, cyclic dimeric guanosine monophosphate (c di-GMP), was recently shown to be bound by stimulator of interferon genes (STING). Binding of c di-GMP by STING activates the Tank Binding Kinase (TBK1) mediated signaling cascades that galvanize the body's defenses for elimination of the pathogen. In addition to c di-GMP, STING has also been shown to function in innate immune responses against pathogen associated molecular patterns (PAMPs) originating from the DNA or RNA of pathogens. The pivotal role of STING in host defense is exemplified by the fact that STING ${ }^{-/}$mice die upon infection by HSV-1. Thus, STING plays an essential role in innate immune responses against pathogens. This opens up an exciting possibility of targeting STING for development of adjuvant therapies to boost the immune defenses against invading microbes. Similarly, STING could be targeted for mitigating the inflammatory responses augmented by the innate immune system. This review summarizes and updates our current understanding of the role of STING in innate immune responses and discusses the future challenges in delineating the mechanism of STING-mediated responses.
\end{abstract}

KEYWORDS innate immune response, adaptor protein, dimeric assembly, crystal structure, c di-GMP

\section{INTRODUCTION}

A number of components of the microbial cell are structurally and chemically different from those found in the cells of the human body. Therefore, microbial intrusion of the human body can be sensed by recognizing such components that are unique to the microbes. Components of the pathogen's cell wall (lipo-polysaccharides, peptidoglycans, carbohydrates), flagella, membrane lipids, nucleic acids, and other virulence factors unique to the pathogen called pathogen associated molecular patterns (PAMPs) are recognized by host's epithelial cells, basophils, eosinophils, neutrophils, phagocytes, mast or natural killer cells at the site of transgression using pattern recognition receptors (PRRs). Real time visuals of the process of microbial invasion by two-photon microscopy confirm the fact that the initial response to microbial invasion by host is directed at localizing the infection (Månsson et al., 2007). Reduction in the vascular flow and clotting are employed to prevent the escape of pathogens as host defense cells infiltrate the site of transgression to eliminate the pathogens. While recognition of PAMPs by sugar binding PRRs like mannose and C-lectin type receptors results in phagocytosis and destruction of the pathogen, sensing of PAMPs by Toll like receptors and cytoplasmic sensors activates signaling cascades resulting in production of interferon (IFN) and cytokines (Takeda et al., 2003). The initial skirmishes results in the spillage of contents of microbial cells and some tissue damage. Both these events result in production of components that have specific molecular patterns that are recognized by the host's innate immune cells (Rubartelli and Lotze, 2007). Recognition sparks a response that initially involves evolutionary conserved innate immune cells, followed by a more precise and elaborate adaptive immune response. For example, sighting of viral RNA in the cytoplasm by RIG-I (retinoic acid inducible gene I) like receptors RIG-I and MDA5 (melanoma differentiation associated gene 5 ) has been shown to activate IRF3 (interferon regulatory factor 3), IRF7 and NF-kB (nuclear factor kappa-light-chain-enhancer of activated $\mathrm{B}$ cells) tran- 
scription factors resulting in production of type I interferon (Kawai and Akira, 2008). Similarly, binding of dsRNA by TLR3 results in recruitment of TRIF. TRIF activates TBK1 and RIP1 kinases leading to transcriptional activation of IFN production via IRF3 and NF-KB (Alexopoulou et al., 2001). These events provide protection against invading microbes.

Amongst the spillage of bacterial contents during the initial encounter of the pathogen with the host defense cells, the secondary messenger molecule, $c$ di-GMP, was recently shown to be bound by a host protein named STING (Burdette et al., 2011). Binding of bacterial c di-GMP by STING activates the TBK1-IRF3/7 signaling cascade, culminating in the production of IFN and cytokines. The truly fascinating aspect of this operation was STING's remarkable ability to discriminate between $\mathrm{c}$ di-GMP and host's nucleotide analogs (Burdette et al., 2011). STING specifically bound c di-GMP and not ATP or GTP. This specificity is beautifully illustrated in the crystal structure of the binary complex of STING with c di-GMP, where the ligand binds in a trough just above the dimer interface (Ouyang et al., 2012). The $c$ di-GMP binding pocket is constrained such that there is no space for accommodating additional phosphate groups of ATP or GTP. In addition to c di-GMP, STING plays an essential role in TLR independent innate immune responses to sighting of DNA and RNA in the cytoplasm (Zhong et al., 2008; Ishikawa et al., 2009; Nakhaei et al., 2010; Prantner et al., 2010; Barber, 2011b; Ishikawa and Barber, 2011). Loss of STING function has been shown to increase susceptibility of cells to microbial infections. Infact, upon infection by HSV-1, STING KO mice died (Ishikawa et al., 2009). Thus, STING plays multiple, essential roles in innate immune signaling. This review discusses our current understanding of the structure, function and regulation of STING in innate immune signaling.

\section{INITIAL CHARACTERIZATION AND LOCALIZATION OF STING}

STING was first discovered in K46 cells as an MHC class II associated protein that participated in the relay of death signals (Jin et al., 2008). STING was essential for ERK mediated, but $\mathrm{c}$ Src independent, apoptosis triggered by MHC class II molecules. Based on the $\mathrm{N}$-terminal sequence consisting of Methionine, Proline, Tyrosine and Serine, the protein was named MPYS. Confocal microscopy of STING-GFP fusion constructs over expressed in K46 cells identified a majority of the protein localized to the mitochondrion (Jin et al., 2008).

During the same time as the publication of the MPYS story, another study appeared in Nature describing the same protein as a STimulator of Interferon Genes (STING) that was essential for innate immune responses (Ishikawa and Barber, 2008). Vesicular stomatitis (VS) and Sendai viral infections were shown to elicit a STING mediated RIG-I dependent, but TLR independent, type I IFN production. Further, STING was shown to participate in innate response to B-DNA via pro- duction of IFN- $\beta$. Both responses involved the participation of TBK1. STING-mediated responses culminated in the activation of NF-KB and IRF3/7 transcription factors. STING co-immuno-precipitated with RIG-I, but not MDA5. In addition, neither poly I:C transfection nor Encephalomyocarditis virus could induce production of IFN- $\beta$ (Ishikawa and Barber, 2008). Taken together, these results suggest that STING participates in RIG-I mediated innate responses, but is not required for MDA5 mediated responses. The localization of STING by this group was predominantly shown to be in the endoplasmic reticulum. Almost simultaneously with the study on STING, came another independent report by Zhong et al. that identified the same protein as Mediator of IRF3 Activation (MITA), which caused activation of IRF-E in 293 cells (Zhong et al., 2008). Cell fractionation and immunoblot analysis revealed that STING localizes to the outer membrane of mitochondrion. This was further confirmed by confocal microscopy of GFP-tagged STING that showed that STING localizes to the mitochondrion. STING associated with VISA at the mitochondrial outer membrane and recruited TBK1 and IRF3. In fact STING acted as a scaffold to enhance interaction of TBK1 with IRF3. Although the association of STING with TBK1 was virus dependant, STING could not interact with IRF3 independently (Zhong et al., 2008).

Further light on the localization of STING was shed by 2 more studies. In one study, using fractionation and fluorescence immuno-staining techniques, Sun et al. (2009) reported that STING resides predominantly in the ER fraction and not mitochondrion. Mutating $\mathrm{R}$ to $\mathrm{A}$ in either of the $2 \mathrm{ER}$ signatures- ${ }^{76} \mathrm{RYR}{ }^{78}$ and ${ }^{178} \mathrm{RIR}{ }^{180}$ —of STING lowered its detection in ER. The role of the RIR signature in ER retention was further confirmed by attaching the sequence to CD8 $\alpha$, which resulted in the change in localization of the protein from cell surface to ER (Sun et al., 2009). Thus, Sun et al. confirmed the localization of STING to ER. Another study conducted by the same Barber group that first identified STING as a potent inducer of IFN, looked at potential localization of STING on the mitochondrion associated membrane (MAM) (Ishikawa et al., 2009). Localization of HA-tagged STING expressed in STING ${ }^{-/}$MEFs was tracked using antibody against HA. The analysis revealed that although STING is predominantly associated with the ER, some STING also got associated with MAMs. On infection of STING HA expressing MEFs with HSV-1 or stimulation by ISD, STING translocated from ER to perinuclear, non-ER microsomal compartments. Such a trafficking of STING could be halted by Brefeldin A, but was unaffected by chloroquine (Ishikawa et al., 2009). This indicates that upon stimulation, STING translocates from ER to vesicles in the perinuclear region via Golgi. Further, STING was shown to associate with Sec5 on stimulation by DNA. TBK1 is also known to associate with Sec5. This was an interesting observation for 2 reasons. Firstly, Sec5 is known to accumulate in perinuclear endosomal compartments (Chien et al., 2006). Secondly, IFN production by Sendai virus involves the RALB and Sec5 
pathways (Ross et al., 2006). This suggests that STING and TBK1 traffic to perinuclear endosomal compartments upon stimulation by B DNA and associate with Sec5. Such an association with Sec5 results in production of type I IFN (Ishikawa et al., 2009). The details on the underlying mechanism for such a production of IFN are not known.

Thus, STING is exclusively found in intra-cellular locations. STING resides on ER, including MAMs. STING participates in RIG-I mediated signaling that involves mitochondrion localized MAVS. Consequently, STING has also been detected at the mitochondrion and has been shown to interact with MAVS. Recently, MAVS residing on the peroxisome was shown to participate in innate immune responses against viruses (Dixit et al., 2010). Thus, it is likely that STING also associates with such a peroxisomal localized MAVS. Upon stimulation by PAMPs like ds DNA, STING migrates to the peri-nuclear vesicles via Golgi. Here, IRF3 and NF-kB are phosphorylated by STING-recruited TBK1, leading to induction of IFN production.

\section{ROLE OF STING IN INNATE IMMUNE RESPONSES}

Viral RNA is sensed by cytosolic RNA helicases containing DExD/H box motifs. So far, three such helicases, RIG-I, MDA5 and laboratory of genetics and physiology 2 (LGP2), that sense RNA with different specificities in the cytosol, have been identified (Jensen and Thomsen, 2012). In addition to the helicases, viral RNA is also sensed by endosomal Toll-like receptors. For example, TLR3 is known to bind double stranded RNA. Similarly, TLRs 7 and 8 bind single stranded RNA (Yoneyama and Fujita, 2010). Sighting of RNA by the receptors initiates a series of events involving adaptor and effector proteins leading to the induction of IFN and IFN inducible genes that provide protection against invading viruses (Yoneyama and Fujita, 2010). Transcription factors like IRF3, IRF7, NF-KB and Stat6 are activated using a set of adaptor proteins and kinases specific to a particular sensor. Some of the kinases play overlapping roles and participate in signaling via more than one sensor. For example, the TANK binding kinase participates in RIG-I as well as TLR3 mediated responses to viral RNA (Yoneyama and Fujita, 2010). STING is known to play an essential role in RIG-I mediated responses against cytosolic RNA (Nakhaei et al., 2010).

In contrast to RNA, detection of DNA as a PAMP by host is poorly understood. TLR9, localized to the endoplasmic reticulum, is known to bind CpG DNA that is mostly found in bacteria and viruses (Kumagai et al., 2008). However, TLR9 deficient mice were able to initiate an innate immune response upon exposure to DNA suggesting existence of additional sensors (Ishikawa et al., 2009). Two distinct types of responses by host to cytosolic DNA have been recorded. A STING-TBK1 mediated response results in production of type I IFNs, while an STING-TBK1 independent, AIM2 inflammasome-mediated response results in IL-1 $\beta$ production (Henao-Mejia et al., 2012). DNA-dependant RNA polymer- ase III, DNA-dependant activator of IFN (DAI) and IFI-16 have also been implicated in sensing DNA. Thus, different types of DNA sensors eliciting distinct responses to sensing of cytosolic DNA exist.

Several lines of evidence suggest that STING participates in innate immune signaling triggered by DNA (Fig. 1) and RNA (Fig. 2) as well as by c di-GMP (Fig. 3) (Bowzard et al., 2009; Barber, 2011a). HEK293T cells do not show detectable expression of STING and therefore are unable to respond to c-di-GMP. Expression of STING, and not I199N mutant of mouse STING could restore the responsiveness of HEK293T cells to c d-GMP (Burdette et al., 2011). To find out if the restored responsiveness was due to binding of STING with $\mathrm{c}$ di-GMP, lysate of HEK293T cells expressing HA-tagged STING was used for cross-linking experiments with radio-labeled $\mathrm{c}$ di-GMP in presence of ultra-violet radiation. A $\sim 40-\mathrm{kDa}$ band corresponding to monomeric STING could be detected in auto-radiographs. Further, immunoprecipitation of STING from HEK293T cell lysates using anti HA antibody and cross-linking of this immuno-precipitated STING with radio-labeled c di-GMP revealed the presence of a $40-\mathrm{kDa}$ band of radio-labeled protein corresponding to STING (Burdette et al., 2011). Unlabeled c di-GMP and not GTP, competed with the labeled c di-GMP for binding with STING. Mutagenesis studies identified key residues involved in binding of $c$ di-GMP. In another recent study, the structure of c di-GMP bound STING (amino acids 139-343) accounted for the observation that dimerization of STING is essential for binding $\mathrm{c}$ di-GMP and not only maps the exact location but also provides information on molecular determinants for specificity in binding c di-GMP (Ouyang et al., 2012). This structural information coupled with mutagenesis and cell based studies firmly establishes STING as a bona fide protein that binds $\mathrm{c}$ di-GMP in vitro as well as in vivo and elicits IFN production.

STING evokes production of IFN in response to transfection by Interferon Stimulatory DNA (ISD), or infections by HSV-1 and Listeria monocytogenes (Barber, 2011b). STING KO mice die upon infection by HSV-1. More recently, a DExDc helicase, DDX41, was shown to sense pathogen derived ds DNA (Zhang et al., 2011). After sensing the ds DNA in myeloid dendritic cells, DDX41 associated with STING and induced IFN I production by activating IRF3/7 and NF-KB. The DEADc domain of DDX41 was shown to bind DNA directly (Zhang et al., 2011). In addition, in vivo binding of DNA by DDX41 was demonstrated using $L$ monocytogenes infected BMDCs as a model. Interestingly, the same DEADc domain that bound DNA was shown to mediate association of DDX41 with STING. Using deletion mapping, the AA 1-154 region of STING was shown to be important for binding DEADc domain of DDX41 and evoking an immune response (Zhang et al., 2011). Thus, STING plays important roles in innate immune responses against cytosolic DNA as PAMPs (Fig. 1). 


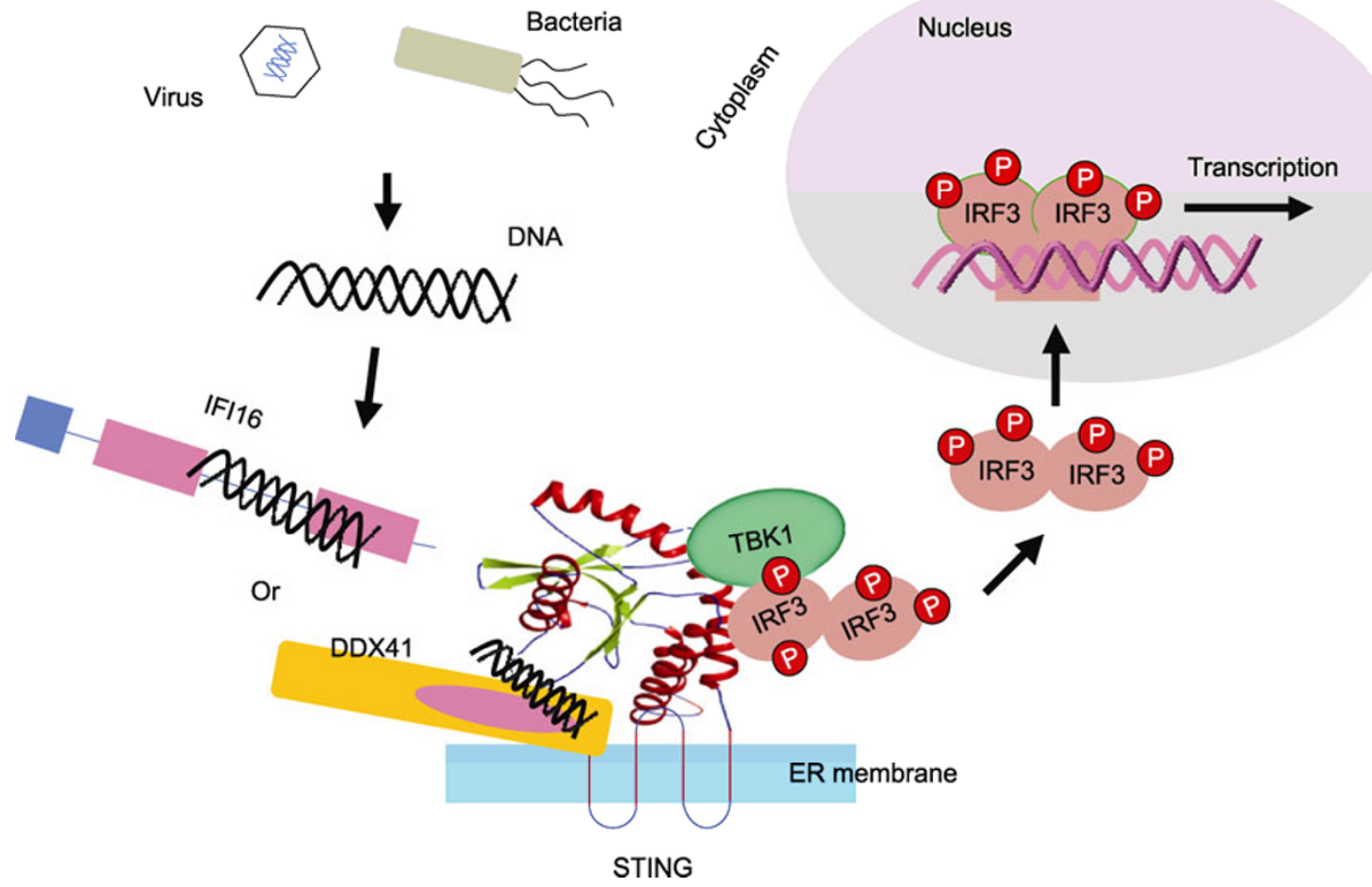

Figure 1. Role of STING in innate immune responses evoked by DNA.

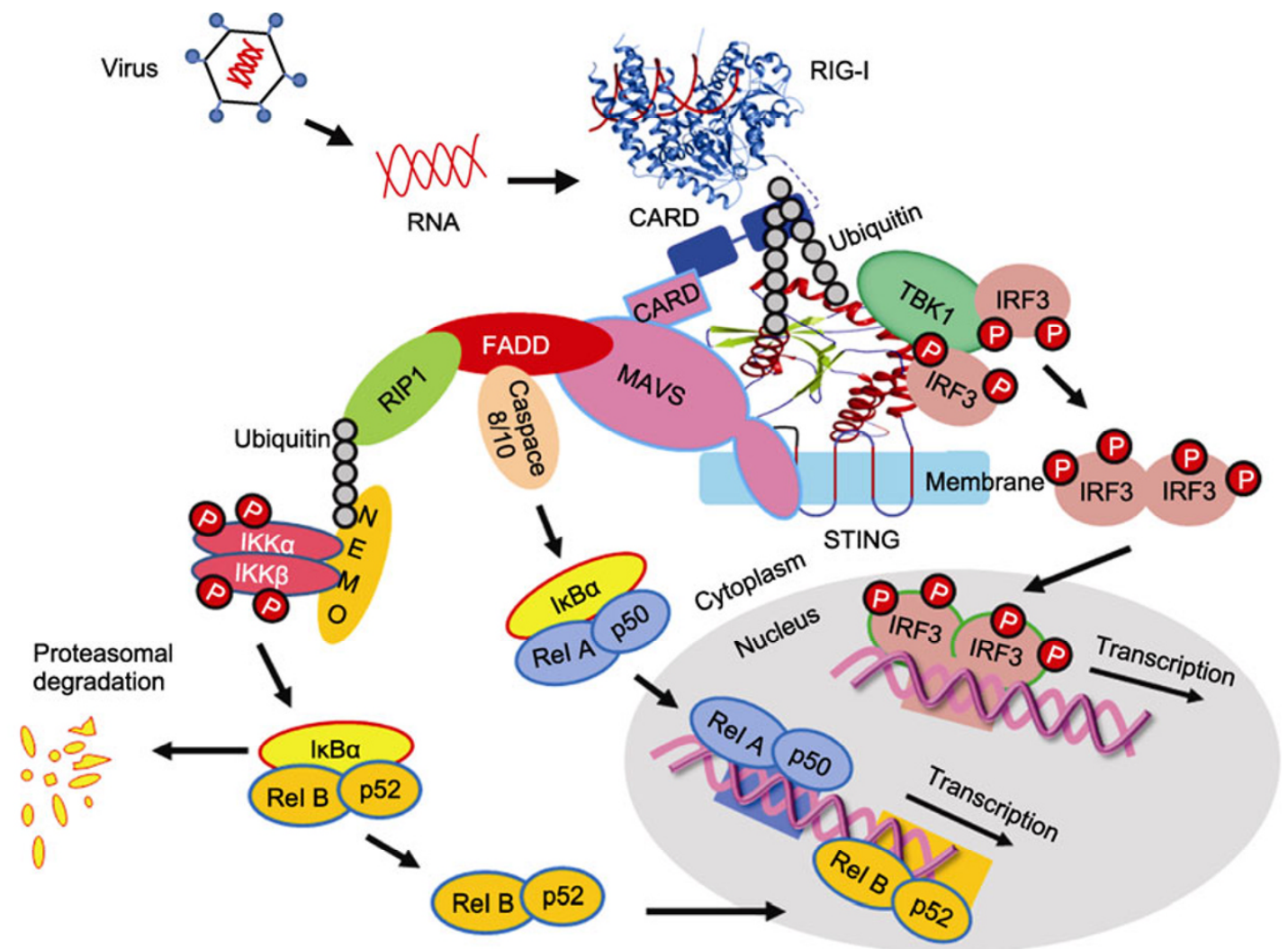

Figure 2. STING participates in innate immune responses triggered by RNA. 


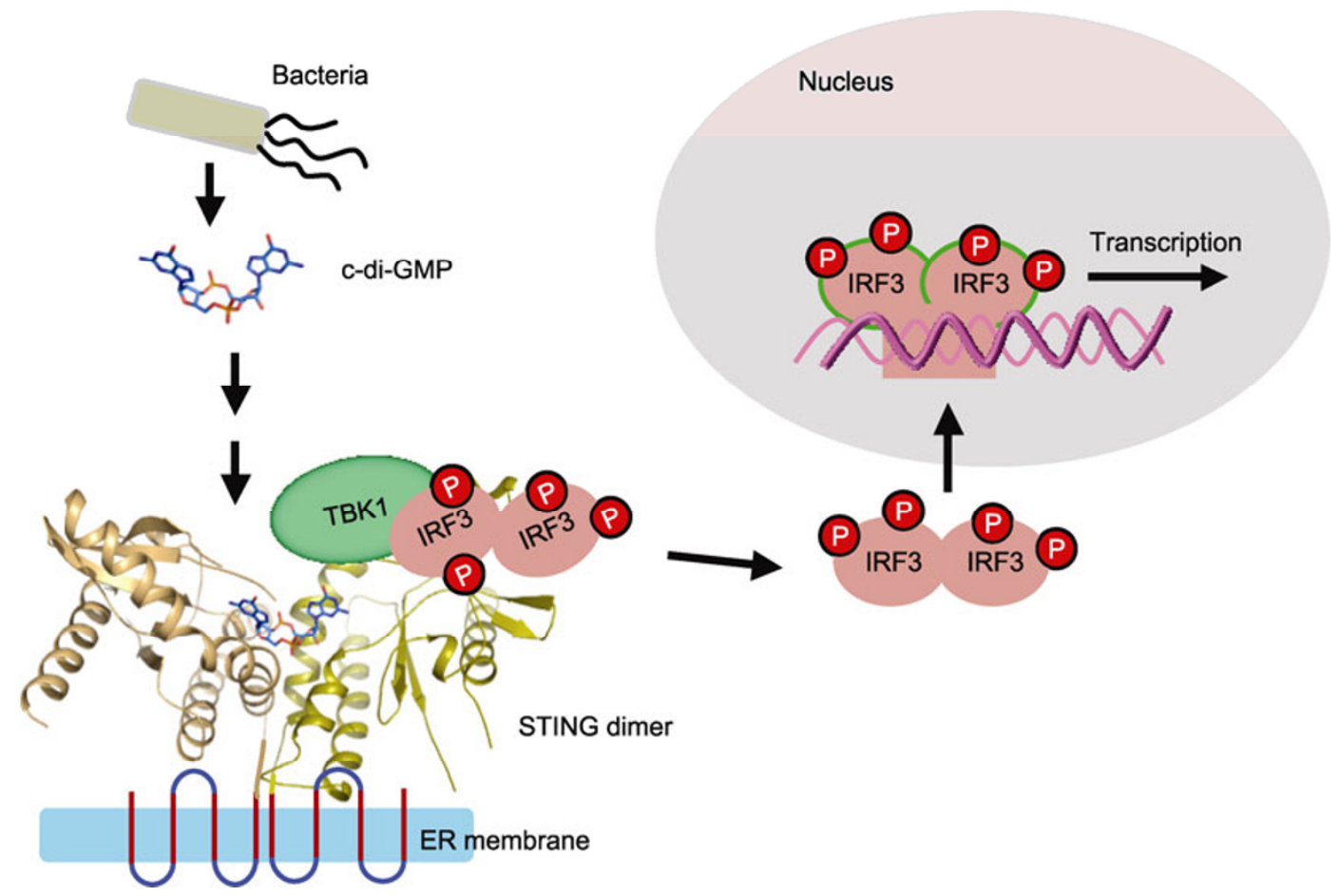

Figure 3. Dimeric STING binds c di-GMP resulting in recruitment and activation of TBK1. TBK1 phosphorylates IRF3, which dimerizes and translocates to the nucleus for transcription of genes.

STING participates in innate immune responses against cytosolic RNA via its association with RIG-I (Nazmi et al., 2012). STING could be specifically immunoprecipitated with RIG-I but not MDA5, after infection of 293T cells with Sendai virus (Ishikawa et al., 2009). The CARD domain of RIG-I (1-184) interacted with STING (Fig. 2). Interestingly, the carboxyl terminal of STING could inhibit the function of RIG-I. STING associated with MAVS at the MAMs or peroxisomes, forms a complex with RIG-I in 293 cells infected with Sendai virus. In a yeast two-hybrid study, STING was shown to associate with TRAPß. Further, in vivo studies using HEK293 cells revealed that STING co-precipitated with TRAP $\beta$ and SEC61 $\beta$ suggesting that STING is present at the site of transfer of the nascent peptide synthesized by ribosomes into the ER by the translocon (Ishikawa et al., 2009). Thus, RIG-I probably senses the translation of viral RNA and activates STING, which recruits TBK1 resulting in induction of IFN production. This is partly supported by a recent report that shows that SEC61 $\beta$ associates with the exocyst complex which recruits and activates TBK1, resulting in production of type I IFN- $\beta$ (Nakhaei et al., 2010).

Although these studies suggest a role for STING in DNA and RNA mediated innate immune signaling (Figs. 1 and 2), there is no direct proof yet of STING's ability to bind DNA or RNA. A structural view of STING bound with DNA/RNA or its complex with any of the sensors (RIG-I, IFI16 or DDX41) bound with DNA/RNA is essential to shed light on whether STING binds nucleic acids directly or indirectly via the formation of a complex with other effector proteins.
Triggers for c di-GMP and nucleic acid mediated activation of STING are located at different regions

Analysis of the structure of STING CTD (AA 139-343) for clues on whether STING can bind DNA or RNA suggests 2 things. First, nucleic acids cannot bind at the same site as $c$ di-GMP. Secondly, if STING were to bind DNA or RNA, this binding would occur at a location remote to the $c$ di-GMP binding site (Fig. 4). Thus, STING might be using different regions of the protein to participate in $c$ di-GMP and DNA/RNA mediated signaling. The support for this hypothesis comes from a R231A mutation of STING that could uncouple STING's c di-GMP signaling from DNA mediated signaling (Burdette et al., 2011). Such a STING mutant could evoke DNA signaling in macrophages derived from the Goldenticket $(g t)$ strain of mice. The $g t$ strains of mice harbor a single nucleotide mutation of STING (T596A) that abolishes expression of the protein and renders the mice incapable of STING mediated signaling (Sauer et al., 2011). Inspection of the structure of the c di-GMP bound STING reveals that R231 (H231 in human STING) is located in vicinity of c di-GMP (Fig. 4). Whether R231 is involved in binding or facilitating docking unliganded as well as liganded structures of STING (Ouyang et al., 2012). However, what is known and can be observed in the structure is that this loop sits right above the c di-GMP and a mutation of the R231A compromises the ability of STING to respond to $c$ di-GMP, but not DNA. Thus, the $c$ di-GMP and nucleic acid signaling might be evoked by different regions of STING (Fig. 4). Further studies like do- 


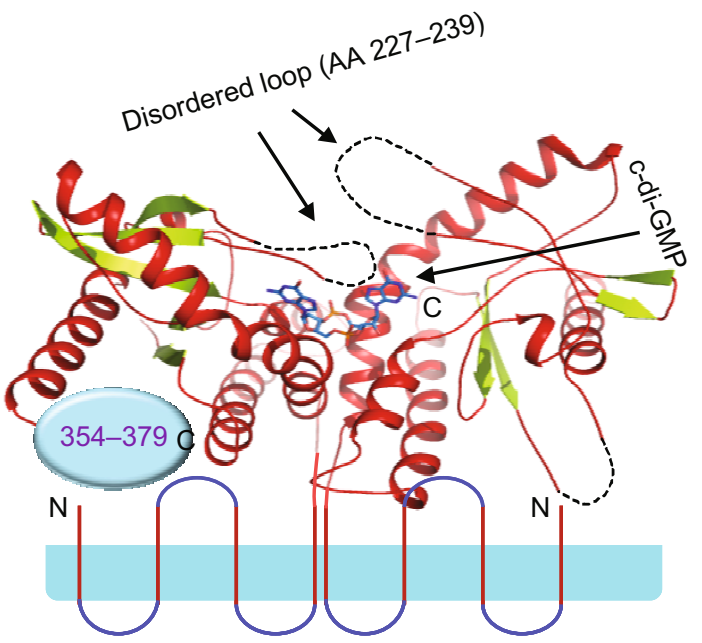

B
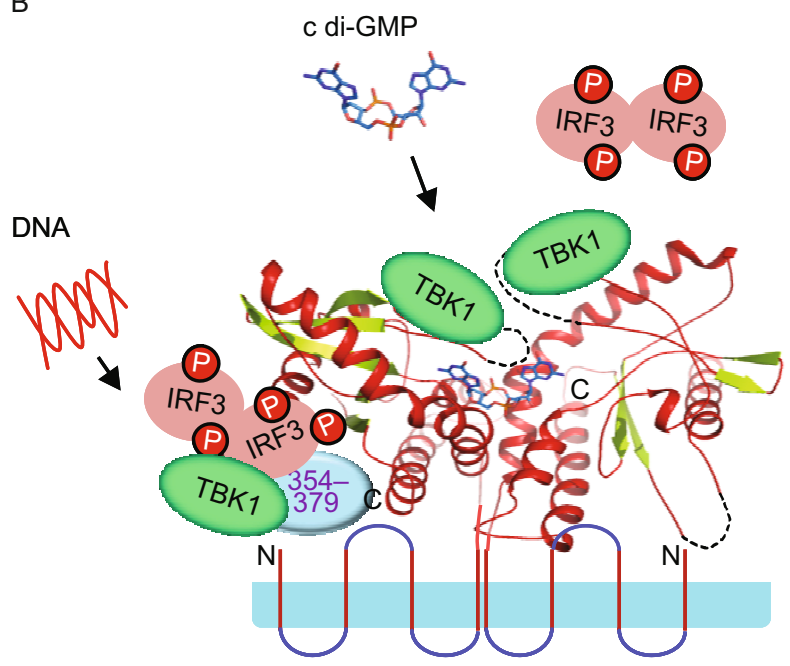

Figure 4. Triggers for activation of innate immune responses by $\mathrm{c}$ di-GMP and nucleic acids reside on distinct regions of STING. A dimer of STING with $\mathrm{N}$ and $\mathrm{C}$ terminals marked is shown in panel $A$ (PDB code 4EF4). The missing 344-379 region of STING is shown as a cyan oval. This region (specifically AA 341-379) binds TBK1 and IRF3 during activation of innate responses by cytoplasmic ds DNA as shown in panel B. The $\mathrm{C}$ di-GMP binding site is remote to this region.

main mapping or a structural view are required to shed more light on these 2 distinct abilities of STING.

The unresolved STING CTD region 344-379, that is not visible in the structure, probably holds the key to answering the question of whether STING can bind nucleic acids. Structures of STING CTD spanning amino acids 139-379, showed a lack of electron density for the terminal 344-379 amino acids (Fig. 4) suggesting that this region is mobile. Alternatively, this region is prone to proteolysis as suggested by the threading results. Threading of the STING AA 344-379 sequence using Pyre server (Kelley and Sternberg, 2009) to obtain clues on the fold reveals nucleic acid binding proteins in the top 4 matches for the prediction. Interestingly, the top match was the C-terminal auto-proteolytic domain of the nuclear pore complex protein nup98, suggesting that the STING AA 344-379 region could possibly be prone to proteolysis. Thus, further studies on the C-terminal end of STING are necessary to clarify the exact role and mechanism of activation of STING in DNA/RNA mediated signaling. In a more recent study, this C-terminal region of STING (AA 281-379) was shown to act as a scaffold between TBK1 and IRF3 (Tanaka and Chen, 2012). Using an in vitro re-constitution system coupled with mutagenesis, Tanaka et al. showed that 39 amino acids at the C-terminus (AA 341-379) are sufficient for activation of TBK1 and phosphorylation of IRF3 in presence of cytosolic double stranded DNA. Mutations that abolished binding of STING to IRF3 could activate TBK1. Further, Ser366 and Leu374 of STING, but not Ser358, are required for phosphorylation of IRF3 (Tanaka and Chen, 2012). However, both these amino acids do not seem to play a role in activation of TBK1. Ser366 was not phosphorylated when STING WT expressed in L929 cells was analyzed by LC-MS.

While our studies on STING show that a truncation of STING without the C-terminal 344-379 amino acids can bind TBK1 and activate production of IFN upon activation by $\mathrm{c}$ di-GMP, Tanaka et al. have concluded that the C-terminal 341-379 amino acids of STING are sufficient for binding TBK1 and activation of IRF3 in presence of ds DNA. Thus, triggers for c-di-GMP and ds DNA mediated immune signaling reside on different regions of STING (Fig. 4). The structural view of c di-GMP bound dimeric STING clearly explains the necessity of AA 152-343 for binding c di-GMP. Similarly, a structural view of the binary or ternary complex of the C-terminal region of STING (AA 341-379) with TBK1 and IRF3 is necessary to shed light on the mechanism of ds DNA mediated activation of STING.

\section{REGULATION OF STING}

STING is subject to direct regulation via at least 2 post-translational modifications-phosphorylation and ubiquitination (Zhong et al., 2008, 2009; Tsuchida, et al., 2010). In addition, proteins involved in autophagy have also been shown to have an impact on the innate immune signaling by STING (Saitoh et al., 2009). More recently, an exo-nuclease, TREX1 has been shown to indirectly regulate the activity of STING (Yan et al., 2010).

Zhong et al. (2008) first reported that phosphorylation of STING Ser358 by TBK1 is critical for activation of IRF-E. STING expressed in 293 cells infected with Sendai virus was readily phosphorylated by TBK1. A STING S358A mutation abolished the ability of STING to interact with TBK1 and the subsequent activation of IRF-E (Zhong et al., 2008). Evidence for an additional effector TBK1 binding site was unveiled in a clever STING-TBK1 GST pulldown study in presence of c di-GMP (Ouyang et al., 2012). Recruitment of TBK1 
was enhanced in presence of c di-GMP. TBK1 binding studies performed in presence of c di-GMP using a C-terminal truncation (STING AA 139-343) revealed the presence of an additional TBK1 binding site that does not involve Ser358, which was previously reported as essential for binding of TBK1 and activation of IRF-E during viral infections. Thus, in presence of c di-GMP, a new TBK1 binding site becomes available and the c di-GMP induced STING-mediated signaling is a result of recruitment of TBK1 at this new binding site. Taken together these results suggest that the triggers for c di-GMP and nucleic acid induced STING-mediated signaling cascades may reside on distinct regions of STING (Fig. 4). Interestingly, so far, only TBK1 has been shown to phosphorylate STING.

Two diagonally opposite effects of the ubiquitination of STING have been reported. A K-48 linked ubiquitination of Lys 150 of STING by RFN5 has been reported to mark STING for proteasomal degradation resulting in down-regulation of the IFN production upon viral infection (Zhong et al., 2009). On the other hand a K-63 linked ubiquitination of the same residue, Lys 150, of STING by TRIM56 was shown to be essential for triggering STING mediated IFN production in presence of dsDNA (Tsuchida et al., 2010; Wang et al., 2011). Such opposite effects of ubiquitination are not surprising because different linkages of ubiquitin are known to encode different messages and are recognized by specific domains. For example, an ubiquitin binding motif of NEMO called UBAN specifically binds K-63 linked ubiquitin and regulates NF-kB signaling (Ikeda et al., 2010). Similarly, K-63 linked ubiquitin chains have also been implicated in repair of damaged DNA. Rap80 specifically binds K-63 linked lysine chains on histones $\mathrm{H} 2 \mathrm{~A}$ and $\mathrm{H} 2 \mathrm{AX}$ and forms a multi protein complex with RING type E3 ligase BRCA1 for repairing double stranded DNA breaks (Ikeda et al., 2010). On the other hand Rpn13, a receptor on the proteasome, specifically recognizes and binds K-48 linked ubiquitin chains (Ikeda et al., 2010). Hence, different linkages of ubiquitin have been known to exert varying physiological responses. Ubiquitin ligase RNF5 interacts with STING in mammalian cells in a virus-dependant manner (Zhong et al., 2009). This interaction is mediated by the transmembrane regions of both the proteins. RNF5 was shown to inhibit Sendai virus induced, as well as poly (I:C) and B-DNA induced activation of IFN- $\beta$ production. This inhibition was shown to be a result of K-48 linked polyubiquitination of Lys150 of STING by RNF5 leading to the proteasomal degradation of STING. Thus, RNF5 down-regulates STING mediated host response upon viral infection. Interestingly, only mitochondrial localized STING was specifically ubiquitinated by RNF5 and not the ER localized STING (Zhong et al., 2009). In another study, TRIM56 ubiquitin ligase was identified as a protein interacting with STING. Specifically, the C-terminal region of STING interacted with the C-terminal of TRIM 56 ligase (Tsuchida, et al. 2010). The ligase carried out K-63 linked polyubiquitination of
Lys150 of STING upon stimulation by dsDNA. This ubiquitination was shown to induce dimerization of STING, which was a pre-requisite for recruitment of TBK1 and downstream signaling (Tsuchida, et al. 2010). Interestingly, TRIM 56 was not found localized to the mitochondrion, but was detected in punctuate structures with STING. Although ubiquitination of K150 of STING by TRIM 56 enhanced the IFN- $\beta$ production in response to stimulation by ds DNA, the mechanism behind this activation is unknown. Since RIG-I was shown to be associated with unanchored K-63 linked ubiquitin chains (Zeng et al., 2010) which caused activation of RIG-I mediated signaling, the polyubiquitination of STING by TRIM 56 is speculated to play a role in the assembly of STING-RIG-I complex via the participation of polyubiquitin chains (Tsuchida, et al. 2010). Thus, depending on the type of poly-ubiquitin linkage, STING mediated signaling could be up or down regulated.

STING seems to be down-regulated by atleast one more mechanism other than ubiquitination-association with auotphagy-related proteins (Saitoh et al., 2009). In ds DNA initiated innate immune responses STING undergoes translocation from the ER to the cytoplasmic punctate structures via Golgi apparatus. Here, STING associates with TBK1 and induces type I interferon production. STING can also associate with autophagy-related proteins. Especially association of STING with ATg9a was shown to abrogate the innate immune response by dsDNA. This down regulation of the innate immune response was independent of autophagy because the puncta enclosing STING did not have the morphological characteristics of autophagosome. Atg9 knockout MEFs showed enhanced association of TBK1 with STING (Saitoh et al., 2009). Thus, in addition to down regulation via ubiquitination, Atg9 and other autophagosome-related proteins seem to negatively regulate STING mediated innate response to dsDNA by an unknown mechanism.

More recently, human immunodeficiency virus (HIV) was shown to employ a cytoplasmic exo-nuclease, TREX1, to evade activation of STING-mediated innate immune signaling (Yan et al., 2010). Once inside the cell, the RNA of HIV is reverse transcribed into DNA and transported to the nucleus for integration into host chromosome. Excess transcripts that are not integrated into the host genome can induce interferon production via the STING-TBK1-IRF3 axis. HIV uses TREX1 to digest the excess transcripts before they can stimulate a STING-mediated innate immune response. Examination of the effect of silencing of various genes on IFN- $\beta$ production in Trex $1^{-/}$MEF cells stimulated by HIV DNA revealed that sensors like TLR9, AIM2, LRRFIP1, HMGB2, and RIG-I or adaptors like MAVS are not involved in HIV-stimulated IFN- $\beta$ production. On the other hand silencing of STING, TBK1 or IRF3 abrogated production of IFN- $\beta$ upon stimulation by HIV (Yan et al., 2010). Thus, TREX1 regulates activation of STING signaling during infection by HIV by degrading the excess DNA transcripts and making them unavailable for 
induction of interferon.

\section{STRUCTURE OF STING, LIGAND BINDING AND SPECIFICITY}

STING is a 379 amino acid long, $42 \mathrm{kDa}$ transmembrane protein (Jin et al., 2008). The N-terminus of STING is predicted to thread the membrane 4 or 5 times, while the C-terminus is cytosolic (Ishikawa and Barber, 2008; Jin et al., 2008; Zhong et al., 2008; Sun et al., 2009). Information on the exact domain boundaries for STING is lacking. Based on the expression and purification of a soluble STING truncation comprising of AA 139-379, the N-terminal transmembrane region of STING has been proposed to span from AA 1-138. The crystal structure of the C-terminal domain of STING (STING CTD; AA 132-343) solved by using bacterially expressed protein, is made up of a single domain with a mixed $\alpha / \beta$ topology (Fig. 5) (Ouyang et al., 2012). A central twisted sheet made up of 5 strands is surrounded by $\alpha$-helices. A proline is seen introducing a kink in the long a5 helix. Such kinks introduced by prolines are commonly observed in membrane proteins and function to introduce mobility in the structure. Although longer constructs (beginning from AA 139) were used for crystallization, only amino acids after 151 were visible in the structure. One explanation for this could be that the region before residue 151 is transmembrane and therefore in absence of a lipid-bilayer, this region is disordered. Similarly, AA 344-379 are not observed in the structure. These amino acids missing at the C-terminus are either prone to degradation or are mobile.

Two structures of STING CTD, unliganded and bound with c di-GMP, were first reported by us (Ouyang et al., 2012). Surprisingly, comparison of the structures reveals no large scale conformational changes upon ligand binding. A loop region strategically located above the $c$ di-GMP binding site is dis-ordered in the unliganded as well as the liganded STING structure (Fig. 4). Because there are no large conformational changes upon binding of $c$ di-GMP, clues to the mechanism of activation of STING by $c$ di-GMP probably reside in this dis-ordered loop (AA 227-239) region. A R231A mutation of mouse STING was shown to abolish its response to $\mathrm{C}$ di-GMP, but such a mutation retained its ability to respond to DNA as a PAMP (Burdette et al., 2011). The inability of this mutation to respond to $c$ di-GMP can be explained by examining the structure, where amino acid 231 is part of the loop sitting above the c di-GMP. Interestingly, R231 is replaced by a histidine in human STING and the implications for the func-
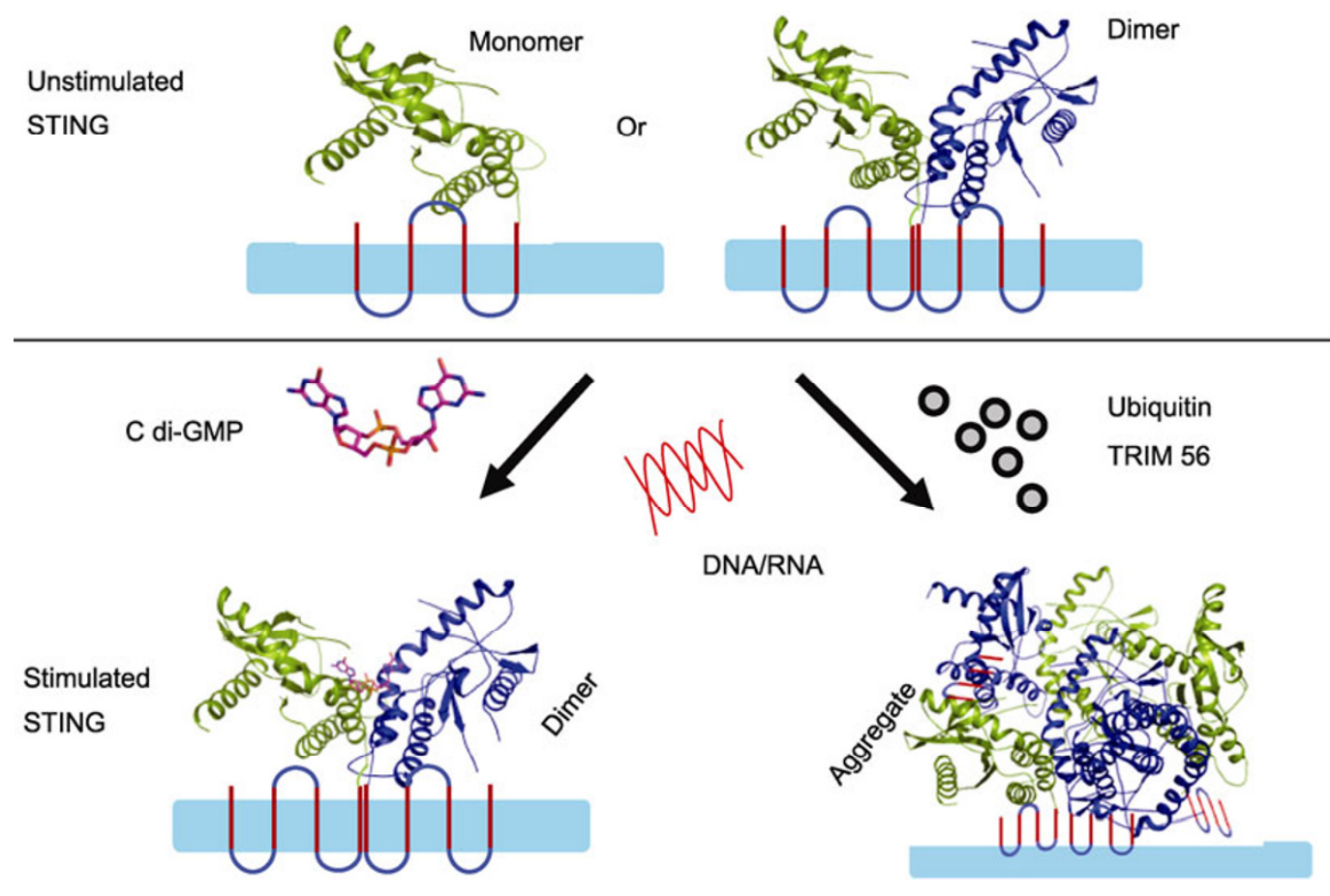

Figure 5. Monomeric as well as dimeric forms of STING have been reported (PDB code 4EF5). Dimerization or aggregation of STING caused by binding of PAMPs or ubiquitin results in its activation.

tion of STING because of this change are currently not known. Binding of c di-GMP has an impact on this loop; possibly rearranging parts of the region around the loop and making it available for binding TBK1 (Fig. 4). This is partly supported by the fact that recruitment of TBK1 was enhanced in presence of c di-GMP. Further, a truncation of STING CTD
(AA 139-344), lacking the Ser358 region, could bind TBK1. Thus, binding of $\mathrm{c}$ di-GMP to STING makes another site available for recruiting TBK1 that activates downstream signaling.

Although both monomers and dimers of STING have been observed in vivo, the oligomerization state of un-stimulated 
STING in vivo is not known (Ishikawa and Barber, 2008; Jin et al., 2008; Sun et al., 2009; Burdette et al., 2011). If un-stimulated STING exists as a monomer in vivo, it is quite likely that $c$ di-GMP could induce its dimerization, resulting in activation of downstream signaling. The crystal structure of STING CTD bound c di-GMP then probably represents one snapshot of STING bound with c di-GMP (Fig. 5). The binding of $c$ di-GMP by STING is specific and the structure can clearly explain why it cannot bind ATP or GTP. There is no space for docking additional phosphate groups in the $c$ di-GMP binding pocket. Studies on binding of $c$ di-AMP, another secondary messenger molecule found in bacteria, to STING seem to indicate that STING may not be able to bind c di-AMP as efficiently as its close homolog c di-GMP. However, it still remains to be determined whether there exists another protein that binds $c$ di-AMP specifically or whether STING was evolved to bind both these secondary messenger molecules. In addition, currently there is no information on the sensor for c di-GMP.

More recently, four different groups independently reported almost identical structures of STING CTD (Huang et al., 2012; Shang et al., 2012; Shu et al., 2012; Yin et al., 2012). Although the structure based conclusions are similar, there is one significant difference at the functional level within these recent reports on STING. Ouyang et al. have reported that the binding of c-di-GMP enhances recruitment of TBK1 and that STING AA 139-344 can recruit TBK1 in presence of c-di-GMP. On the contrary, Yin et al. found no interaction between STING CBD (c-di-GMP binding domain; AA 139-343) and TBK1 either in presence or absence of c-di-GMP (Yin et al., 2012). The authors postulate that upon binding C-di-GMP, the C-terminal tail domain (CTT; AA 340-379) is released from its intra-molecular interaction with STING CBD; resulting in activation of STING-mediated signaling. Shu et al. (2012) have concluded that rates of phosphorylation of STING by TBK1 and the subsequent dimerization of IRF3 were not affected by c-di-GMP.

\section{STING FUNCTIONS AS A DIMER}

Jin et al. first noticed that most of the STING exists as a dimer in cells (Jin et al., 2008). Although, Jin et al. reported the presence of dimers of STING in cells, Sun et al., showed that dimerization of STING was essential for production of type I IFN (Sun et al., 2009). Over expression of TBK1 in 293 cells resulted in hyper-phosphorylation and dimerization of STING. By domain mapping, they showed that the dimerization was mediated by the $\mathrm{N}$-terminal transmembrane region. Further, the requirement of dimerization of STING for induction of IFN was demonstrated by employing a coumermycin-induced dimerization assay (Farrar et al., 1996). One molecule of coumermycin is known to bridge 2 molecules of DNA GyrB. By constructing a chimera containing the C-terminal of STING fused to GyrB, a dose dependant courmermycin induced dimerization of the chimera was shown to result in an increase in the IFN- $\beta$ production. Thus, dimerization of STING induces IFN production.

The first structural evidence for existence of STING dimers came via the $2.45 \AA$ and $2.15 \AA$ resolution crystal structures of unliganded and c di-GMP bound STING CTD, respectively. One molecule of c di-GMP binds 2 molecules of STING (Burdette et al., 2011). This is clearly seen in the structure of c di-GMP bound STING (Fig. 6). The structure of c di-GMP bound STING explains why a dimer of STING is essential for binding $\mathrm{c}$ di-GMP. The $\mathrm{c}$ di-GMP binding pocket is formed by participation of 2 molecules of STING (Fig. 6). Although the structural and functional evidence for a role of dimerization of STING in activation of innate immune signaling is compelling, the exact mechanism underlying the dimerization is still not clear. There are 2 very different explanations for dimerization of STING. Tucsida et al., have shown that K-63 linked ubiquitination of STING K150 by TRIM 56 results in dimerization or oligomerization of STING, which was a pre-requisite for the activation of STING (Fig. 5) (Tsuchida et al., 2010). On the other hand our structure-function studies reveal that dimerization of STING CTD is signal independent. STING uses dimerization as a means to bury a hydrophobic path (AA 152-173) and that the dimerization of STING is neither initiated by $\mathrm{c}$ di-GMP nor ubiquitination. Bacterially expressed STING CTD already exists as a dimer in solution.

Interestingly, the crystal structure of STING CTD reveals an unexpected location for the dimer interface (AA 153-173) because it seems to suggest that STING's dimer interface is not part of its transmembrane region. The interface is dominated by hydrophobic amino acids that mediate the dimerization. The physiological relevance of the dimer interface and the requirement of dimerization of STING for IFN production were verified by selecting the amino acids observed in the formation of the dimer interface and mutating them in order to break the dimer interface. 293T cell based luciferase reporter assays as a measure of IFN- $\beta$ induction upon activation of STING by B-DNA were used to evaluate the effect of the mutations in full length STING. Simultaneously, the same mutations performed in STING CTD were expressed in E coli to monitor the oligomerization state of these mutants. Information from these two experiments was expected to reveal whether the effect of the mutations was because of a breakdown of the overall secondary structure of STING or disruption of the dimer interface. Four mutations of amino acids located at the dimer interface of full length STING-V155R, W161A, Y164A, and I165R-lost their ability to induce interferon production, while a G158L mutation showed highly diminished activity. Amongst all the mutants, only I165R and G158L could be expressed as soluble protein in $E$ coli. However, both these mutant proteins were highly aggregated, when produced and purified from $E$ coli. Further, in co-immuno-precipitation assays performed on protein expressed in 293T cells, the G158L mutant failed to interact with itself or STING WT, suggesting a defect in dimerization 
A

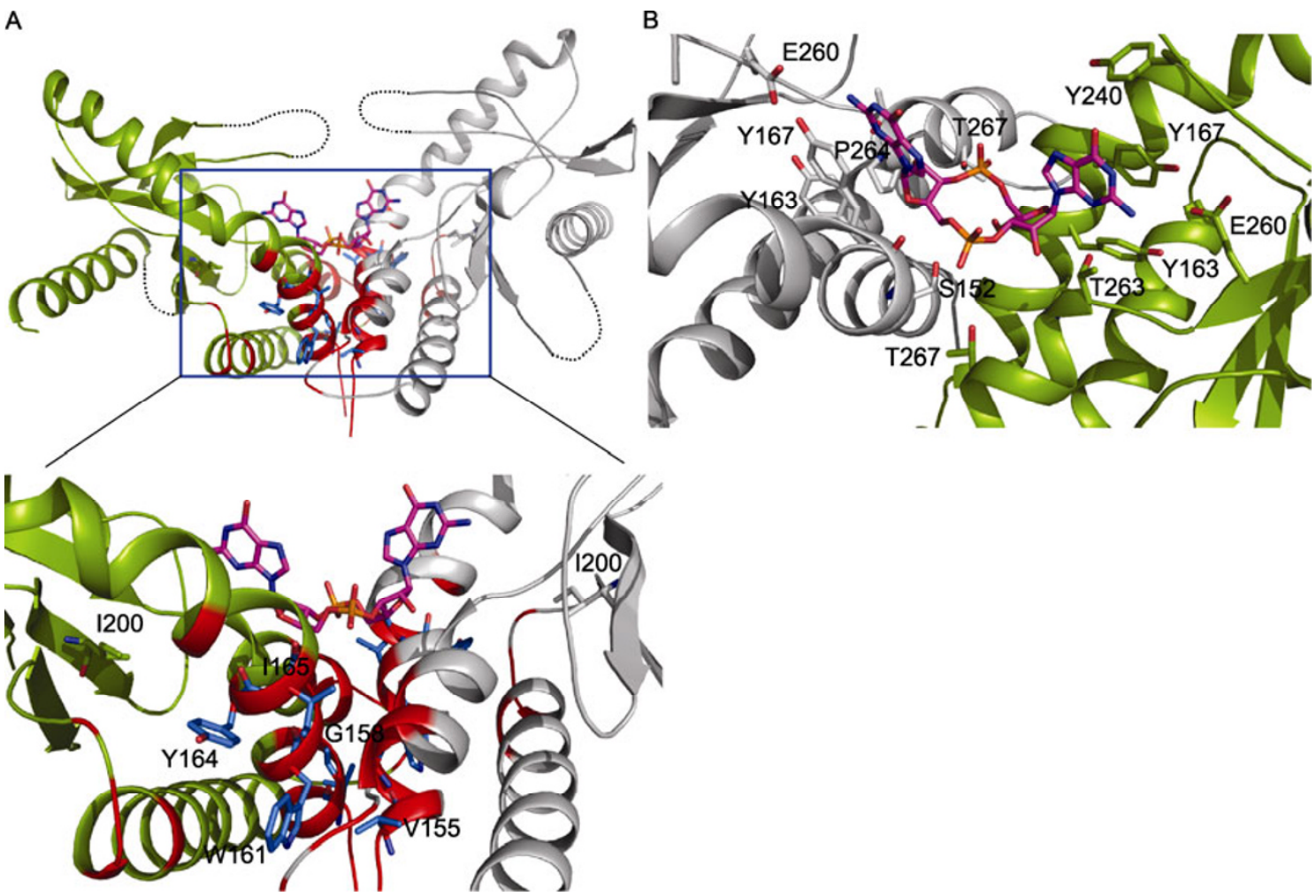

Figure 6. A dimer of STING binds one molecule of $\mathrm{c}$ di-GMP. (A) The formation of $\mathrm{c}$ di-GMP binding pocket requires the participation of 2 monomers of STING (colored grey and green). The dimer interface is colored in red. Amino acids mutated to break the dimer interface (Ouyang et al., 2012) are shown as blue sticks. The gt strain of mouse harbors an I199N mutation that abolishes STING mediated response to c di-GMP. Location of this mutation (I200 in human STING) is shown. (B) Residues interacting with c di-GMP (magenta sticks) are shown as green or grey colored sticks in panel B. Atomic co-ordinates of PDB file with accession code $4 \mathrm{EF} 4$ were used to generate the figures.

that abrogated the IFN production. This seems to support the fact that the dimer interface observed in the structure is physiologically relevant and could represent one of the conformations assumed by STING in vivo. The importance of this dimer interface in inducing interferon production under real-time scenarios of bacterial infections is highlighted by the docking of $c$ di-GMP into the trough of the dimer interface (Fig. 6). When the STING CTD protein was mixed with c di-GMP, crystallized, and the structure of the complex was solved, $c$ di-GMP was observed sitting in the pocket, just above the dimer interface formed by 2 monomers of STING. Thus, a dimer of STING is required to bind $\mathrm{c}$ di-GMP and initiate the downstream signaling.

Most of the studies on STING have reported a monomeric species of STING in vivo in addition to the STING dimer (Fig. 6) (Ishikawa and Barber, 2008; Jin et al., 2008; Zhong et al., 2008). Therefore, there exists an alternative mechanism, other than dimerization, by which the hydrophobic patch observed at the dimer interface in the structure of STING CTD is buried. It is possible that, prior to dimerization, STING is kept monomeric via interactions with the $\mathrm{N}$-terminal transmembrane region. Alternatively, STING may be kept mono- meric via its interactions with VISA or TBK1 or other known or currently unknown interacting proteins. Needless to say, the nature of the interactions holding STING in a monomeric state is probably weak and permits dimerization. In addition, the dis-ordered loop might act as a spacer to further decrease the strength of these interactions. Upon binding a PAMP directly, example c di-GMP, or indirectly via RIG-I and IFI16, STING probably undergoes a conformational change resulting in dimerization or oligomerization. A posttranslational modification like ubiquitination could also possibly induce such an oligomeric transition of STING. The dimerization of STING observed in the presence of PAMP c di-GMP, neatly captured in the crystal structure, leads to activation of downstream signaling. However, whether dimerization occurs before binding of the ligand (as observed in the structure of unliganded STING CTD) or after receiving a signal from c di-GMP in full length STING within the lipid bi-layer environment of the membrane and the exact mechanism of dimerization of STING are still unknown. In addition, whether STING is able to reverse the dimerization or oligomerization is also currently unknown. Further, the question of how is the signal or c di-GMP sensed and transferred to 
STING remains unanswered. Since STING could bind $c$ di-GMP directly, it is plausible that STING functions as a sensor or an adaptor depending on the location of the encounter with the pathogen. In the latter case, the identity of the sensor is unknown.

\section{INTERACTION OF STING WITH STAT6}

Recently, STAT6 was shown to be recruited by STING during virus induced innate immune responses (Chen et al., 2011). Upon RNA or DNA viral infections, Stat6 co-localizes with STING in an IL-4/13 independent manner. The DNA binding domain (AA 260- 430) of STAT6 and the C-terminal region (AA 178-379) of STING were shown to be sufficient for the interaction of STAT6 with STING. Phosphorylation of Ser407 of STAT6 by TBK1 associated with STING preceded the subsequent phosphorylation of Stat6 Y641, which results in homodimerization of Stat6, nuclear translocation and transcription of specific genes. Such activation of STAT6 was observed in JAK deficient cells and also cells treated with JAK inhibitors, suggesting existence of a separate, JAK-independent STAT6 signaling cascade (Chen et al., 2011). This is further supported by uncoupling of the virus mediated activation of STAT6 from IL-4 mediated activation by a L551A mutation of STAT6. The Y641 phosphorylation and subsequent dimerization of STAT6 upon viral infection was impaired in the L551A mutant. However, such a mutant retained the IL-4 mediated activation of STAT6. Surprisingly, activation of STAT6 via STING resulted in transcription of a different set of genes than those observed in the canonical JAK-STAT pathway. CCL2, CCL20 and CCL26 chemokines that attract various immune cells were transcribed upon activation of STAT6 recruited by STING. Consequently, Stat6 ${ }^{-1}$ mice exhibit greater susceptibility to viral infections due to impaired production of chemokines and recruitment of immune cells. Interestingly, the STING dependent STAT6 signaling does not evoke activation of neither NF-kB nor IRF3 transcription factors that are normally activated by the STING-TBK1-IRF3/7 pathway. Thus, the STING-STAT6 signaling increases the repertoire of innate immune signaling responses to DNA and RNA viruses. While infection by DNA viruses activates STING, which recruits STAT6 and TBK1, resulting in the dimerization of STAT6, nuclear translocation and production of the STAT6 transcribed proteins, infection by RNA viruses involves the participation of MAVS that resides either on mitochondrial associated ER membrane or peroxisome associated membranes. Activation of STING by RNA probably leads to the formation of a large complex containing STAT6-STING-MAVS-TBK1 that phosphorylates STAT6, leading to transcription of STAT6 dependant genes. Interestingly, although STAT6 does not interact directly with TBK1 or MAVS, both proteins are essential for STING activated STAT6 mediated innate responses to RNA viruses (Chen et al., 2011). The identity of the tyrosine kinase that phosphorylates Y641 of STAT6 essential for its activation as an effector in STING dependant innate immune responses is not known. Knowledge of the nature of the tyrosine kinase could further shed light on the convergence and the subsequent divergence of STING and STAT6 signaling.

Research during the past 5 years has firmly established STING as a critical component of the innate immune signaling. STING plays a central role in TLR-independent response to ds DNA, RIG-I dependant response to RNA and the innate immune response to the sighting of $c$ di-GMP. Interestingly, all these STING-mediated responses evoke the phosphorylation, dimerization and activation of transcription factors using kinases like TBK1 recruited by STING. Thus, STING plays a role in converging signaling initiated by PAMPs as diverse as bacterial secondary messenger molecules like $c$ di-GMP with signaling activated by DNA or RNA associated PAMPs. Such a central role for STING in innate immune responses makes it an attractive target for therapeutic interventions, including development of vaccacines and adjuvants to boost the immune system for the prevention of infections. Further, STING could be targeted for subduing inflammatory responses arising out of innate immune signaling. In this context, the structure of STING CTD and the available functional data seem valuable for guiding the development of STING-targeted therapies. It would be interesting to see how STING performs during evaluation of its potential for therapeutic interventions. Clearly, several opportunities and niches exist to modulate specific aspects of STING mediated responses catering to either PAMPs originating from DNA/RNA or c di-GMP and other yet to be discovered signals. For example, the c di-GMP binding site could be targeted for specifically modulating a response to microbial, especially bacterial intrusions. Similarly, the dimer interface could be targeted for abrogating dimerization using small molecules. Preventing access to K150 could offer yet another level of control over STING mediated responses. An understanding of the outcome of such modulations would not only shed more light on mechanism of STING mediated responses, but also help evaluate the therapeutic potential of small molecules targeting STING.

\section{ACKNOWLEDGEMENTS}

This work was supported by the National Natural Science Foundation of China (Grant No. 31070660) and the Ministry of Science and Technology of China (No. 2009DFB30310).

\section{ABBREVIATIONS}

AIM 2, absent in melanoma 2; c di-GMP, cyclic dimeric guanosine monophosphate; CCL2, chemokine (C-C motif) ligand 2; CD8a, cluster of differentiation 8, alpha chain; DAI, DNA-dependant activator of IFN; DDX41, DEAD box polypeptide 41; ER, endoplasmic reticulum; LPS, lipo-polysaccharides; HSV, herpes simplex virus; IFI16, $\gamma$-interferon inducible protein Ifi-16; IFN, interferon; IRF3, interferon regulatory factor 3 ; ISD, interferon stimulatory DNA; KO, knock out; LGP2, laboratory of genetics and physiology 2; MAVS, 
mitochondrial antiviral signaling protein; MDA5, melanoma differentiation associated gene 5; MHC, major histocompatibility complex; NF-KB, nuclear factor kappa-light-chain-enhancer of activated B cells; PAMP, pathogen associated molecular pattern; PRR, pattern recognition receptor; RIG-I, retinoic acid inducible gene I; RIP1, receptor-interacting serine/threonine-protein kinase 1; RNF5; ring finger protein 5, E3 ubiquitin ligase; SEC61, protein transport protein SEC61 subunit; STAT6, signal transducer and activator of transcription; STING, stimulator of interferon genes; TRAP, translocon associated protein; TBK1, tank binding kinase; TLR, toll-like receptor; TRIF, TIR-domain-containing adapter-inducing interferon- $\beta$; TRIM56, tripartite motif-containing protein 56; VISA, virus-induced signaling adapter

\section{REFERENCES}

Alexopoulou, L., Holt, A.C., Medzhitov, R., and Flavell, R.A. (2001). Recognition of double-stranded RNA and activation of NF-[kappa]B by Toll-like receptor 3. Nature 413, 732-738.

Barber, G.N. (2011a). Cytoplasmic DNA innate immune pathways. Immunol Rev 243, 99-108.

Barber, G.N. (2011b). Innate immune DNA sensing pathways: STING, AIMII and the regulation of interferon production and inflammatory responses. Curr Opin Immunol 23, 10-20.

Bowzard, J.B., Ranjan, P., Sambhara, S., and Fujita, T. (2009). Antiviral defense: RIG-Ing the immune system to STING. Cytokine Growth Factor Rev 20, 1-5.

Burdette, D.L., Monroe, K.M., Sotelo-Troha, K., Iwig, J.S., Eckert, B., Hyodo, M., Hayakawa, Y., and Vance, R.E. (2011). STING is a direct innate immune sensor of cyclic di-GMP. Nature 478, 515-518.

Chen, H., Sun, H., You, F., Sun, W., Zhou, X., Chen, L., Yang, J., Wang, Y., Tang, H., Guan, Y., et al. (2011). Activation of STAT6 by STING is critical for antiviral innate immunity. Cell 147 , 436-446.

Chien, Y., Kim, S., Bumeister, R., Loo, Y.-M., Kwon, S.W., Johnson, C.L., Balakireva, M.G., Romeo, Y., Kopelovich, L., Gale Jr, M., et al. (2006). RalB GTPase-mediated activation of the Ik-B family kinase TBK1 couples innate immune signaling to tumor cell survival. Cell 127, 157-170.

Dixit, E., Boulant, S., Zhang, Y., Lee, A.S.Y., Odendall, C., Shum, B., Hacohen, N., Chen, Z.J., Whelan, S.P., Fransen, M., et al. (2010). Peroxisomes are signaling platforms for antiviral innate immunity. Cell 141, 668-681.

Farrar, M.A., Alberola-lla, J., and Perlmutter, R.M. (1996). Activation of the Raf-1 kinase cascade by coumermycin-induced dimerization. Nature 383, 178-181.

Henao-Mejia, J., Elinav, E., Strowig, T., and Flavell, R.A. (2012). Inflammasomes: far beyond inflammation. Nat Immunol 13, 321-324.

Huang, Y.-H., Liu, X.-Y., Du, X.-X., Jiang, Z.-F., and Su, X.-D. (2012). The structural basis for the sensing and binding of cyclic di-GMP by STING. Nat Struct Mol Biol 19, 728-730.

Ikeda, F., Crosetto, N., and Dikic, I. (2010). What determines the specificity and outcomes of ubiquitin signaling? Cell 143, 677-681.

Ishikawa, H., and Barber, G.N. (2008). STING is an endoplasmic reticulum adaptor that facilitates innate immune signalling. Nature
455, 674-678.

Ishikawa, H., and Barber, G.N. (2011). The STING pathway and regulation of innate immune signaling in response to DNA pathogens. Cell Mol Life Sci 68, 1157-1165.

Ishikawa, H., Ma, Z., and Barber, G.N. (2009). STING regulates intracellular DNA-mediated, type I interferon-dependent innate immunity. Nature 461, 788-792.

Jensen, S., and Thomsen, A.R. (2012). Sensing of RNA viruses: a review of innate immune receptors involved in recognizing RNA virus invasion. J Virol 86, 2900-2910.

Jin, L., Waterman, P.M., Jonscher, K.R., Short, C.M., Reisdorph, N.A., and Cambier, J.C. (2008). MPYS, a novel membrane tetraspanner, is associated with major histocompatibility complex class II and mediates transduction of apoptotic signals. Mol Cell Biol 28, 5014-5026.

Kawai, T., and Akira, S. (2008). Toll-like receptor and RIG-1-like receptor signaling. Ann N Y Acad Sci 1143, 1-20.

Kelley, L.A., and Sternberg, M.J.E. (2009). Protein structure prediction on the Web: a case study using the Phyre server. Nat Protoc 4, 363-371.

Kumagai, Y., Takeuchi, O., and Akira, S. (2008). TLR9 as a key receptor for the recognition of DNA. Advanced Drug Delivery Reviews 60, 795-804.

Lu, Y.-C., Yeh, W.-C., and Ohashi, P.S. (2008). LPS/TLR4 signal transduction pathway. Cytokine 42, 145-151.

Månsson, L.E., Melican, K., Boekel, J., Sandoval, R.M., Hautefort, I., Tanner, G.A., Molitoris, B.A., and Richter-Dahlfors, A. (2007). Real-time studies of the progression of bacterial infections and immediate tissue responses in live animals. Cell Microbiol 9, 413-424.

Nakhaei, P., Hiscott, J., and Lin, R. (2010). STING-ing the antiviral pathway. J Mol Cell Biol 2, 110-112.

Nazmi, A., Mukhopadhyay, R., Dutta, K., and Basu, A. (2012). STING mediates neuronal innate immune response following Japanese Encephalitis Virus infection. Sci Rep 2, 347.

Ouyang, S., Song, X., Wang, Y., Ru, H., Shaw, N., Jiang, Y., Niu, F., Zhu, Y., Qiu, W., Parvatiyar, K., et al. (2012). Structural analysis of the STING adaptor protein reveals a hydrophobic dimer interface and mode of cyclic di-GMP binding. Immunity 36, 1073-1086.

Prantner, D., Darville, T., and Nagarajan, U.M. (2010). Stimulator of IFN gene is critical for induction of IFN-beta during Chlamydia muridarum infection. J Immunol 184, 2551-2560.

Ross, C., Hatzoglou, A., Parrini, M.-C., White, M.A., Chavrier, P., and Camonis, J. (2006). RalB mobilizes the exocyst to drive cell migration. Mol Cell Biol 26, 727-734.

Rubartelli, A., and Lotze, M.T. (2007). Inside, outside, upside down: damage-associated molecular-pattern molecules (DAMPs) and redox. Trends Immun 28, 429-436.

Saitoh, T., Fujita, N., Hayashi, T., Takahara, K., Satoh, T., Lee, H., Matsunaga, K., Kageyama, S., Omori, H., Noda, T., et al. (2009). Atg9a controls dsDNA-driven dynamic translocation of STING and the innate immune response. Proc Natl Acad Sci U S A 106, 20842-20846.

Sauer, J.D., Sotelo-Troha, K., von Moltke, J., Monroe, K.M., Rae, C.S., Brubaker, S.W., Hyodo, M., Hayakawa, Y., Woodward, J.J., Portnoy, D.A., et al. (2011). The N-ethyl-N-nitrosourea-induced Goldenticket mouse mutant reveals an essential function of Sting 
in the in vivo interferon response to Listeria monocytogenes and cyclic dinucleotides. Infect Immun 79, 688-694.

Shang, G., Zhu, D., Li, N., Zhang, J., Zhu, C., Lu, D., Liu, C., Yu, Q., Zhao, Y., Xu, S., et al. (2012). Crystal structures of STING protein reveal basis for recognition of cyclic di-GMP. Nat Struct Mol Biol 19, 725-727.

Shu, C., Yi, G., Watts, T., Kao, C.C., and Li, P. (2012). Structure of STING bound to cyclic di-GMP reveals the mechanism of cyclic dinucleotide recognition by the immune system. Nat Struct Mol Biol 19, 722-724.

Sun, W., Li, Y., Chen, L., Chen, H., You, F., Zhou, X., Zhou, Y., Zhai, Z., Chen, D., and Jiang, Z. (2009). ERIS, an endoplasmic reticulum IFN stimulator, activates innate immune signaling through dimerization. Proc Natl Acad Sci U S A 106, 8653-8658.

Takeda, K., Kaisho, T., and Akira, S. (2003). Toll-like receptors. Annu Rev Immunol 21, 335-376.

Tanaka, Y., and Chen, Z.J. (2012). STING Specifies IRF3 Phosphorylation by TBK1 in the cytosolic DNA signaling pathway. Sci Signal 5, ra20.

Tsuchida, T., Zou, J., Saitoh, T., Kumar, H., Abe, T., Matsuura, Y., Kawai, T., and Akira, S. (2010). The ubiquitin ligase TRIM56 regulates innate immune responses to intracellular double-stranded DNA. Immunity 33, 765-776.

Wang, J., Liu, B., Wang, N., Lee, Y.M., Liu, C., and Li, K. (2011). TRIM56 is a virus- and interferon-inducible E3 ubiquitin ligase that restricts pestivirus infection. J Virol 85, 3733-3745.

Yan, N., Regalado-Magdos, A.D., Stiggelbout, B., Lee-Kirsch, M.A., and Lieberman, J. (2010). The cytosolic exonuclease TREX1 inhibits the innate immune response to human immunodeficiency virus type 1. Nat Immunol 11, 1005-1013.

Yin, Q., Tian, Y., Kabaleeswaran, V., Jiang, X., Tu, D., Eck, M.J., Chen, Z.J., and Wu, H. (2012). Cyclic di-GMP sensing via the innate immune signaling protein STING. Mol Cell 46, 735-745.

Yoneyama, M., and Fujita, T. (2010). Recognition of viral nucleic acids in innate immunity. Rev Med Virol 20, 4-22.

Zeng, W., Sun, L., Jiang, X., Chen, X., Hou, F., Adhikari, A., Xu, M., and Chen, Z.J. (2010). Reconstitution of the RIG-I pathway reveals a signaling role of unanchored polyubiquitin chains in innate immunity. Cell 141, 315-330.

Zhang, Z., Yuan, B., Bao, M., Lu, N., Kim, T., and Liu, Y.J. (2011). The helicase DDX41 senses intracellular DNA mediated by the adaptor STING in dendritic cells. Nat Immunol. 12, 959-965.

Zhong, B., Yang, Y., Li, S., Wang, Y.Y., Li, Y., Diao, F., Lei, C., He, X., Zhang, L., Tien, P., et al. (2008). The adaptor protein MITA links virus-sensing receptors to IRF3 transcription factor activation. Immunity 29, 538-550.

Zhong, B., Zhang, L., Lei, C., Li, Y., Mao, A.P., Yang, Y., Wang, Y.Y., Zhang, X.L., and Shu, H.B. (2009). The ubiquitin ligase RNF5 regulates antiviral responses by mediating degradation of the adaptor protein MITA. Immunity 30, 397-407. 